\title{
Social Support and Participation as Factors Relating to Ikigai and Life Satisfaction in Lonely Older Japanese
}

\author{
Ai Fukuzawa ${ }^{1,2}$ (1) $\cdot$ Ikuko Sugawara ${ }^{3}$
}

Accepted: 6 February 2022

(c) The Author(s) 2022

\begin{abstract}
This study investigated associations among loneliness, social support, social participation, and well-being among the Japanese elderly. We predicted that the negative association between loneliness and well-being would be weaker in people with adequate social support and frequent social participation. We measured ikigai and life satisfaction as indices of well-being. Ikigai and life satisfaction both include satisfaction with a person's current and past life, yet ikigai also includes unique concepts such as satisfaction with social interactions and positive expectations for the future. Data of 418 Japanese aged 75 and older were analyzed; findings demonstrated that loneliness was negatively related to ikigai but not life satisfaction. There was a significant interaction between loneliness and social support for life satisfaction and ikigai. The interaction between loneliness and the frequency of social participation was significant only for ikigai. Post-hoc analysis indicated that social support and social participation frequency were negatively related to the negative association between loneliness and well-being, especially ikigai. These results suggest that $i k i$ gai and life satisfaction have a differential relationship to loneliness and social interaction because the concept of ikigai uniquely included perceived social roles.
\end{abstract}

Keywords Ikigai $\cdot$ Life satisfaction · Life worth living $\cdot$ Loneliness $\cdot$ Social participation $\cdot$ Social support

Ai Fukuzawa

fukuzawa.ai@gmail.com

1 Institute for Future Initiatives, The University of Tokyo, Bldg.8.,7F 7-3-1 Hongo, Bunkyo-ku, Tokyo 113-8656, Japan

2 Japan Society for the Promotion of Science, Kojimachi Business Center Building, 5-3-1 Kojimachi, Chiyoda-ku, Tokyo 102-0083, Japan

3 Present Address: Department of Service Management, Bunri University of Hospitality, 311-1 Kashiwabara-Shinden Sayama-shi, Saitama 350-1336, Japan 


\section{Introduction}

Loneliness is a subjective emotion experienced when the quality or the quantity of interpersonal relationships does not fulfill a person's needs (Asher \& Weeks, 2013). Research has shown that loneliness has negative associations with several types of well-being across all age groups. Loneliness is related to high depression (Cacioppo et al., 2006), low quality of life (QOL: Lim \& Kua, 2011), low life satisfaction (Goodwin et al., 2001; Mellor et al., 2008), and low psychological well-being (Bhagchandani, 2017). Although previous studies have investigated methods of reducing loneliness through interventions, these studies have shown contradictory results regarding the efficacy of loneliness interventions (Gardiner et al., 2018). Thus, we suggest that not only developing interventions to reduce loneliness itself, but also identifying the factors that could prevent loneliness from reducing people's well-being is essential. The current study investigated whether social support and social participation frequency are related to the association between loneliness and well-being among Japanese people aged 75 and older.

Although people experience loneliness across all age groups, it has been demonstrated that the association between age and loneliness is a U-shaped so that loneliness tends to decrease between young adulthood and early old age and increase again in very old age (Demakakos et al., 2006; Pinquart \& Sorensen, 2001). Later old age, such as 75 years and older, is when people's well-being may worsen. It has been demonstrated that ikigai, one of the indices of well-being, is negatively related to age among the elderly (Kondo \& Kamada, 2004) and tends to be lower among individuals 75 years and older than those in early old age (Kokubo et al., 2014). Moreover, it was demonstrated that loneliness increases mortality risk through lowered well-being among people in later old age (Wang et al., 2020). Increased loneliness and decreased well-being in later old age might be critical risk factors for mortality. Nevertheless, only a few studies have investigated loneliness and well-being in people of this age group, and only a handful of these studies have examined factors that might decrease the negative association between loneliness and well-being among them.

We suggest that variables related to social interactions, such as social support and social participation, would decrease the negative association between loneliness and well-being among people aged 75 and older. There are two reasons why we focus on social support and social participation. First, the social buffering hypothesis (Cohen \& Wills, 1985) posits that social support weakens the negative relationship between the experience of stressful events and well-being. Being lonely should be a stressful state; therefore, loneliness would be negatively related to well-being. However, according to the social buffering hypothesis, we can suggest that individuals in later old age with adequate social support can maintain their well-being, even if they experience high levels of loneliness. Second, along with social support, social participation is also said to be a crucial aspect of social interaction variables (Harper, 2002; Putnam, 1993). Moreover, it has been suggested that neighborhood social participation opportunities are 
crucial for well-being, especially for older adults who spend most of their time in their community (Zhang et al., 2018). It is possible that social support and social participation are essential especially for people in later old age, in order to protect their well-being from stressful state such as high loneliness.

Indeed, previous studies have suggested that social support and social participation might decrease the negative association between loneliness and several types of well-being in different age groups. Perceived social support mediated the association between loneliness and depression among Chinese people aged 60 and older, suggesting that social support is "a potential protective factor for lonely elderly persons" (Liu et al., 2016; p 756). It was also shown that social support partially mediated the negative association between loneliness and life satisfaction among early adolescents (Kapikiran, 2013). Regarding social participation, a study of very lonely people aged 44 to 98 years demonstrated that the frequency of participation in leisure activities was positively related to life satisfaction and psychological well-being (Kim et al., 2017), although this study targeted only those with high loneliness.

The previous studies cited above, however, are not limited to people aged 75 and older. As mentioned, loneliness tends to increase with age (e.g., Pinquart \& Sorensen, 2001), and loneliness results in mortality risk (Wang et al., 2020), especially in later old age. Therefore, it is essential to examine whether social support and social participation reduce the negative relationship between loneliness and well-being among those aged 75 and older. Our study is a cross-sectional study so that we cannot predict a causal association in which quantity of social support and frequency of social participation would weaken the negative relationship between loneliness and well-being. However, we can predict that the negative relationship between loneliness and well-being should be weaker among those with adequate social support or frequent social participation than in those with less social support and social participation among those in later old age.

Moreover, many previous studies on well-being have mainly used measures and terms developed in Western countries, such as depression and life satisfaction. It has been demonstrated that the meaning of well-being is different across cultures (Uchida \& Ogihara, 2012) so that it is essential to focus on "non-Western wellbeing related concepts" (Kono \& Walker, 2020, p. 102). Therefore, this study treated both life satisfaction and ikigai as indices of well-being. Ikigai, which is translated as "life worth living," is one of the most commonly used indices of well-being in older Japanese adults. Ikigai is defined as the feeling experienced by a person when doing helpful things for another person or society; thus, the person engaging in these actions would feel that life is worth living (Nakanishi, 1999; Shibata, 1998). The concepts of ikigai and other types of well-being, such as life satisfaction, commonly include the satisfaction with a person's current and past life. In contrast, the concept of ikigai uniquely includes a person's satisfaction with his or her social interactions and positive expectations for the future (Imai et al., 2009). Ikigai is also explained as a combination of general well-being and the feeling of having social roles (Shibata, 2002).

Well-being has been categorized into two aspects: hedonic well-being, consisting of a high degree of pleasure and a low degree of displeasure, and eudaimonic wellbeing, consisting of a perceived purpose in life and meaning of life (Ryan \& Deci, 
2001). Many types of well-being are classified as either hedonic (e.g., life satisfaction, mood) or eudaimonic well-being (e.g., psychological well-being). However, it has been suggested that ikigai represents both hedonic and eudaimonic aspects of well-being (Kumano, 2018; Sone et al., 2008; Tanno et al., 2009). As the concept of ikigai and the other types of well-being are somewhat different, the patterns among loneliness, social interaction variables, and well-being might also differ according to the type of well-being. Nevertheless, few studies have assessed several different types of well-being and tested relationships among well-being, loneliness, and variables related to social interaction within the same survey. Therefore, this study assessed two types of well-being, ikigai, and life satisfaction, and compared their relationships with variables related to loneliness and social interactions.

Several studies have demonstrated that maintaining life satisfaction and ikigai decreases mortality risk (Koivumaa-Honkanen et al., 2000; Tanno et al., 2009). Therefore, maintaining life satisfaction and ikigai among Japanese in later old age is an essential concern. Although life satisfaction and ikigai are similar, a demonstrated difference between them is that ikigai tends to decline with age, which is not the case for life satisfaction (Kobe Active Aging Research Hub, 2017). As far as we know, the current study is the first to investigate whether social support and social participation are related to the negative association between loneliness and two aspects of well-being, life satisfaction, and ikigai, among Japanese people aged 75 and older. We expect that this study would expand the understanding of wellbeing among adults in this age group.

\section{Hypotheses}

This study assumed that loneliness and ikigai and loneliness and life satisfaction would be weakly related among people with adequate social support and frequent social participation. Thus, we test the interaction effect between loneliness and social support and the interaction between loneliness and social participation on $i k i$ gai and life satisfaction.

Previous studies have demonstrated that social support was negatively related to the strength of the relationship between loneliness and several types of well-being, including life satisfaction among adolescents (Kapikiran, 2013) and depression among people aged 60 and older (Liu et al., 2016). Although these studies were not limited to those aged 75 and older, we assumed that these findings would be shown among Japanese late-stage older adults. Thus, we predict that the negative relationship between loneliness and life satisfaction would be weaker among those with adequate social support than among those with inadequate social support.

Moreover, one notable feature of ikigai is that it represents both hedonic and eudaimonic aspects of well-being (e.g., Kumano, 2018). In a study targeting people experiencing loneliness, frequent participation in leisure activities was positively related to psychological well-being, an index of eudaimonic well-being (Kim et al., 2017). Because the study of Kim et al (2017) targeted only lonely people, their findings are interpreted as indicating the interaction effect between loneliness and social participation frequency on eudaimonic well-being. Thus, 
because ikigai represents eudaimonic well-being, we expect that the interaction between frequency of social participation and loneliness would be significant on ikigai. Moreover, actively participating in social activities is more proactive than receiving social support. We assumed that proactive activities such as social participation are necessary to fulfill a person's perception of having social roles, which is one of the unique aspects of ikigai, even if he or she is subject to loneliness. Therefore, we predict that the negative association between loneliness and ikigai would be weaker among those with frequent social participation.

On the other hand, few studies have investigated whether social support decreased the relationship between loneliness and ikigai. Moreover, because the concepts of life satisfaction do not include the feeling that one has social roles, social participation might not be related to the negative relationship between loneliness and life satisfaction, unlike ikigai. However, previous studies have demonstrated that ikigai and life satisfaction have similar relationships with the other variables (Pinquart \& Sorensen, 2000; Fujimoto et al., 2004), so that it is possible that the relational patterns among social support, social participation, and well-being are similar to those between ikigai and life satisfaction. Therefore, we do not make a specific hypothesis about the differences in relational patterns between life satisfaction and ikigai. We developed the following two hypotheses about the interaction between loneliness and social support, social participation, on well-being.

(1) The negative relationship between loneliness and well-being (life satisfaction and ikigai) would be weaker among people with more social support than people with less social support.

(2) The negative relationship between loneliness and well-being (life satisfaction and ikigai) would be weaker among people with more frequent social participation than those with less frequent social participation.

\section{Method}

\section{Participants}

We analyzed data from a survey conducted in Kashiwa city, a town in Tokyo's suburbs. All the district residents who were 12 years and older were requested to return the completed questionnaires by post. Approximately 2700 questionnaires were distributed, and 1249 were returned. We analyzed the data of people who were 75 years and older $(\mathrm{N}=418 ; 225$ men and 183 women, mean age $=$ 79.06 years, $S D=3.57$ ) because this study targeted people in that age group. The survey was conducted according to the Declaration of Helsinki. Informed consent was obtained from all participants before they participated in the study. This study was conducted after receiving the Life Science Research Ethics and Safety approval from the ethical committee of The University of Tokyo. 


\section{Measures}

Life satisfaction. Life satisfaction was assessed by one item stating, "Overall, I am satisfied with my current life" The participants responded to this item using a 5-point scale ranging from 1 (agree) to 5 (disagree). This item has been repeatedly used in surveys conducted in Japan, including the Japanese General Social Survey and The Public Opinion Survey on the Life of the People conducted by the Cabinet Office, and several previous studies (Cheung \& Lucas, 2014; Kobau et al., 2010). The score was reverse-coded.

Ikigai. Ikigai was assessed by one item stating, "I have enough ikigai, and I also have ease of mind and willpower." The participants responded using a 5-point scale ranging from 1 (agree) to 5 (disagree). This item has been repeatedly used in the Japanese Value Orientations, a longitudinal survey conducted by NHK Broadcasting Culture Research Institute. The score was reverse-coded.

Loneliness. Loneliness was assessed using a direct measure of loneliness, "I feel isolated from others," which was extracted from the UCLA loneliness scale (Russel et al., 1978). This item has been repeatedly used in major social surveys, including ELSA (English Longitudinal Study of Ageing), HRS (Health and Retirement Study), and the Japanese Aging and Health Dynamics Study. The participants responded using a 5-point scale ranging from 1 (agree) to 5 (disagree). The score was reverse-coded.

Frequency of social participation. The frequency of social participation was assessed by inquiring, "How often do you go out for recreational and social activities?" Participants responded using a 5-point scale ranging from 1 (less than once a month) to 5 (more than three times a week).

Social support. Social support was assessed by asking if the participants knew of anyone to which they could (i) make requests when leaving home for an extended period, including checking in on the home and watering plants or tending to a garden; (ii) ask for help in case of disaster or emergency; and (iii) visit and talk about problems; in their neighborhood. The participants responded 1 (yes) or 0 (no) to these items. We treated the total number of responses with 1 (yes) as the social support score.

Demographic variables. We assessed age, sex $(1=$ male; $2=$ female $)$, marital status $(1=$ married; $-1=$ not married $)$, educational level $(1=$ junior high school to 4 = university of graduate school), work status ( 1 = working; $-1=$ not working), economic satisfaction and health status. Economic satisfaction was assessed by the item "I am satisfied with my ability to make ends meet and my economic situation." The participants responded to these questions using a 5-point scale ranging from 1 (agree) to 5 (disagree). The health status was assessed by inquiring, "How healthy do you think you are?', The responses were scored using a 5-point scale ranging from 1 (very healthy) to 5 (not healthy). The scores were reverse-coded. 


\section{Results}

Table 1 shows descriptive statistics of all study variables. Among the study variables that we included in the analyses of this study, the missing data of social support (43 cases; $10.29 \%$ ) and social participation (100 cases; $23.92 \%$ ) accounted for more than $5 \%$ of all cases. We conducted a missing value analysis in SPSS version 28 . Those with missing data in social support were older $(t=2.13, p<0.05)$ and less healthy $(t=-2.98, p<0.01)$ than those without missing data in this variable. Those with missing data in social participation were older $(t=5.46, p<0.01)$, less healthy $(t=$ $-5.14, p<0.01)$, had lower educational levels $(t=-2.04, p<0.05)$, higher loneliness $(t=2.87, p<0.01)$, and lower ikigai $(t=-3.54, p<0.01)$ than those without missing data in this variable.

Table 2 shows correlations between the variables. It can be seen that economic satisfaction, health status, and the frequency of social participation were positively correlated, and loneliness was negatively correlated with life satisfaction and ikigai.

Table 1 Descriptive statistics of study variables

\begin{tabular}{llccc}
\hline & & $N$ & $\%$ or mean & $S D$ \\
\hline Life satisfaction & & 406 & 4.01 & 0.89 \\
Ikigai & & 402 & 3.64 & 1.02 \\
Age & & 418 & 79.06 & 3.57 \\
Gender & Males & & & \\
& Females & 225 & 55.10 & \\
Marriage & & 183 & 44.90 & \\
& Married & & & \\
Education & Not married & 317 & 75.80 & \\
& & 101 & 24.20 & \\
& Junior high school & & & \\
& High school & 15 & 3.67 & \\
Junior college & 135 & 33.01 & \\
Work status & University or graduate school & 65 & 15.89 & \\
& & & 47.43 & \\
Economic satisfaction & Working & 34 & 8.10 & \\
Health status & Not working & 384 & 91.90 & \\
Loneliness & & 407 & 3.90 & 0.93 \\
Social Support & & 411 & 3.47 & 1.08 \\
Frequency of social participation & 406 & 2.00 & 0.96 \\
\hline & & 375 & 1.51 & 1.15 \\
& & 318 & 2.88 & 1.28 \\
\hline
\end{tabular}

Life satisfaction, ikigai, economic satisfaction, health status, loneliness, and frequency of social participation scores ranged from 1 to 5 . Social support scores ranged from 0 to 3 


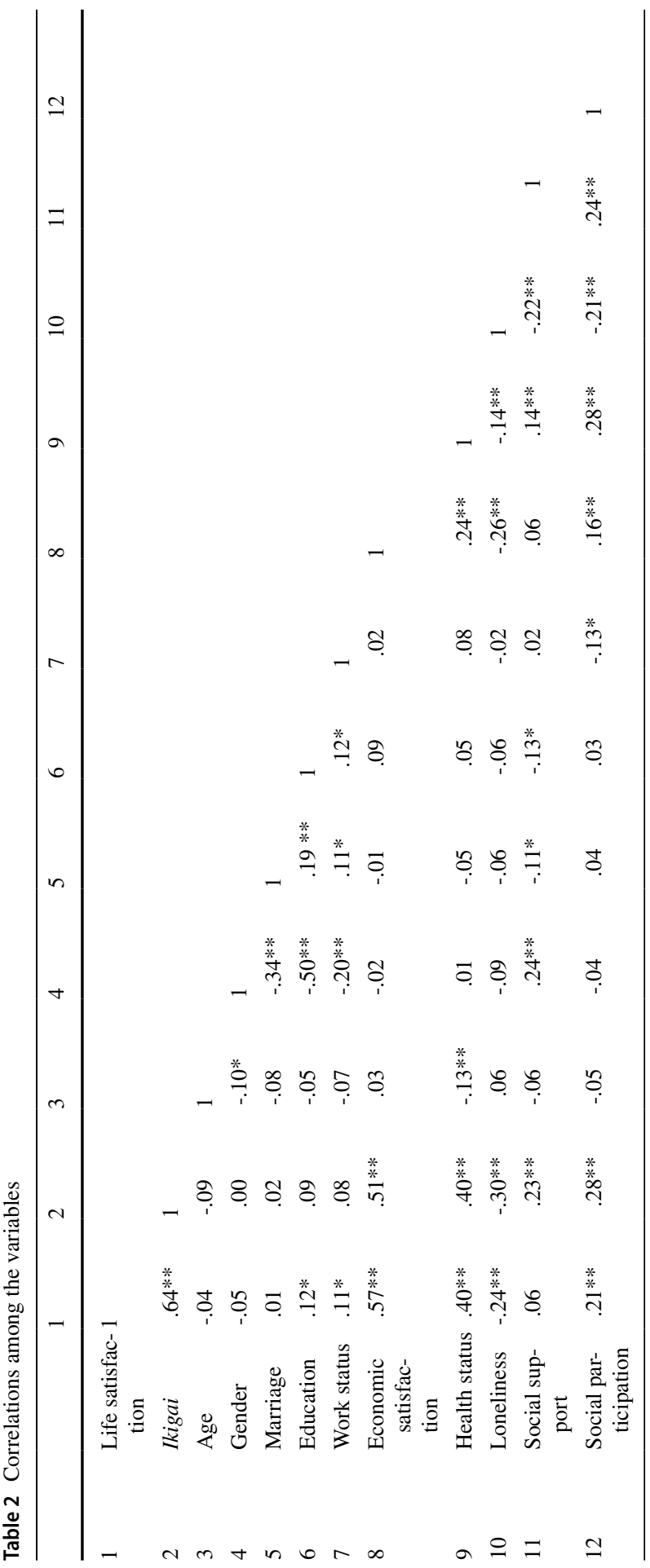


Moreover, educational level and work status were positively correlated with life satisfaction, whereas social support was positively correlated with ikigai.

The results of multiple regression analyses for predicting life satisfaction and ikigai, from loneliness, social support, frequency of social participation, and demographic variables, are shown in Tables 3 and 4. In Model 1, we inputted the independent variables by using the forced entry method. Results indicated that economic satisfaction and health status were positively related to life satisfaction and ikigai (Tables 3 and 4). It can be seen that social support is positively related to ikigai, whereas loneliness is negatively related to ikigai (Table 4). Then, we included the interaction terms for loneliness and social support and the frequency of social participation in Model 2 (Tables 3 and 4). The interaction terms were developed by multiplying the centered variables, and each interaction term was inputted consecutively. The results indicated that the interactional effect of loneliness and social support on life satisfaction (Table 3) and ikigai (Table 4) was significant. The interaction between loneliness and the frequency of social participation in ikigai was also significant (Table 4). However, the interaction between loneliness and the frequency of social participation in life satisfaction was not significant (Table 3 ). ${ }^{1}$

A simple slopes posthoc analysis was conducted for significant interactional terms based on previous studies (e.g., Cohen et al., 2002). We compared the relationship between loneliness and life satisfaction between participants with more $(+1 S D)$ and less social support $(-1 S D)$. Results indicated that loneliness was negatively associated with life satisfaction $(t=-3.61, p<0.01)$ in participants with less social support $(-1 S D)$, whereas it was not significantly related to life satisfaction $(t$ $=-0.24$, n.s.) in participants with more social support $(+1 S D)$ (Fig. 1). Moreover, loneliness was negatively associated with ikigai among people with less social support $(t=-5.56, p<0.01)$ and less frequent social participation $(t=-5.13, p<0.01)$. In contrast, these associations were not significant among people with more social support $(t=-1.18$, n.s. $)$ or those with more social participation $(t=-1.64$, n.s. $)$ (Figs. 2 and 3).

\footnotetext{
${ }^{1}$ Of the 418 participants, 133 had missing data. We conducted multiple imputation procedures for the missing data with SPSS version 28, which created 20 imputed data files. Multilevel regression analyses were conducted for the original data and the 20 imputed data files. The significance of the statistical values of the regression models based on the original data without missing values $(n=285)$ and the pooled statistical values of the regression models based on the multiple imputed files were broadly consistent with one exception: although the interaction between loneliness and social participation on life satisfaction was not significant $(B=0.05$, n.s. $)$ on the original data, its interaction was significant according to the pooled statistical value (The pooled $B=0.09, p<.01$ ). Similar to the other significant interaction effects, loneliness was negatively related to life satisfaction only among those with less social participation, whereas its negative relationship was not significant among those with more social participation. However, life satisfaction did not have a direct negative relationship with loneliness both in the original data and in the imputed data. Thus, our conclusion that social buffering hypothesis is more consistent with the findings regarding ikigai than findings regarding life satisfaction is not altered even if the interaction between loneliness and social participation on life satisfaction is significant. Moreover, the significance of the other statistical values was the same between the original data and the imputed data. Thus, this study shows the analysis results on the original data without missing values.
} 
Table 3 Results of the multiple regression analysis predicting life satisfaction

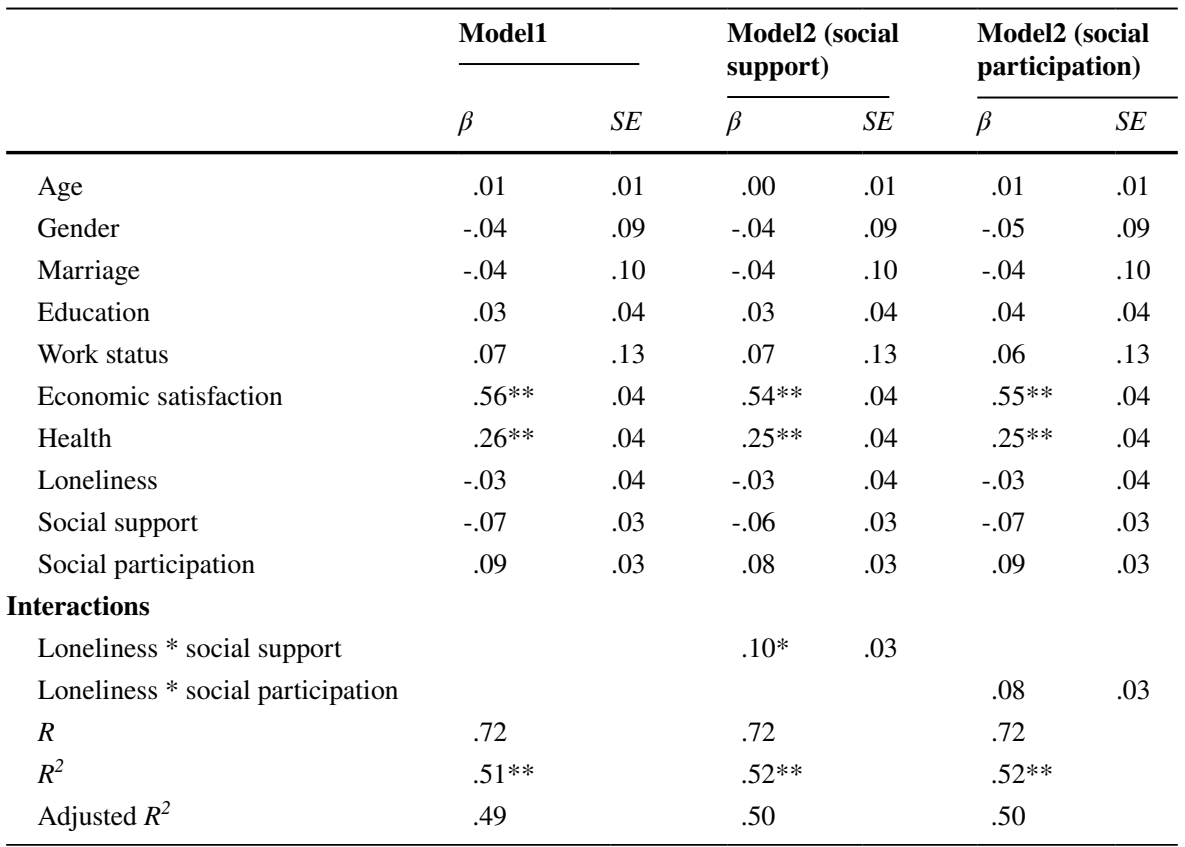

${ }^{*} p<.05 * * p<.01$

\section{Discussion}

We examined whether social support and social participation would be related to the negative association between loneliness and ikigai and the relationship between loneliness and life satisfaction among Japanese people aged 75 and older. Previous studies have demonstrated that loneliness is a risk factor for older people's wellbeing; however, only a few studies have identified factors related to the negative association between loneliness and well-being among people aged 75 and older.

The results demonstrated that ikigai and life satisfaction were positively correlated with each other. However, the multilevel regression analysis results showed a significant negative association between loneliness and ikigai, whereas loneliness was not significantly related to life satisfaction. Moreover, Ikigai was positively associated with social support, whereas there was no significant association between social support and life satisfaction. These findings suggest that ikigai had stronger associations with variables related to interpersonal relationships such as loneliness and social support than life satisfaction had.

Moreover, the results of the interactions between loneliness and social interaction variables indicated that the negative association between loneliness and ikigai was weaker among those with adequate social support and frequent social participation than those with inadequate social support and less social participation, which supported Hypotheses 1 and 2 of this study. Because this study is cross-sectional, we cannot determine a causal effect. However, we suggest that 
Table 4 Results of the multiple regression analysis predicting ikigai

\begin{tabular}{|c|c|c|c|c|c|c|}
\hline & \multicolumn{2}{|l|}{ Model1 } & \multicolumn{2}{|c|}{$\begin{array}{l}\text { Model2 (social } \\
\text { support) }\end{array}$} & \multicolumn{2}{|c|}{$\begin{array}{l}\text { Model2 (social } \\
\text { participation) }\end{array}$} \\
\hline & $\beta$ & $S E$ & $\beta$ & $S E$ & $\beta$ & $S E$ \\
\hline Age & -.03 & 0.01 & -.05 & .01 & -.04 & .01 \\
\hline Gender & .01 & 0.11 & .01 & .11 & .00 & .11 \\
\hline Marriage & .05 & 0.12 & .06 & .12 & .06 & .12 \\
\hline Education & .06 & 0.05 & .06 & .05 & .07 & .05 \\
\hline Work status & .04 & 0.16 & .04 & .16 & .04 & .16 \\
\hline Economic satisfaction & $.48^{* *}$ & 0.05 & $.47 * *$ & .05 & $.47 * *$ & .05 \\
\hline Health & $.20 * *$ & 0.05 & $.19^{* *}$ & .05 & $.18 * *$ & .05 \\
\hline Loneliness & $-.13 * *$ & 0.05 & $-.13 * *$ & .05 & $-.13 * *$ & .05 \\
\hline Social support & $.14^{* *}$ & 0.04 & $.15^{* *}$ & .04 & $.14 * *$ & .04 \\
\hline Social participation & .08 & 0.04 & .07 & .04 & .08 & .04 \\
\hline \multicolumn{7}{|l|}{ Interactions } \\
\hline Loneliness * social support & & & $.10^{*}$ & .04 & & \\
\hline Loneliness * social participation & & & & & $.12 * *$ & .03 \\
\hline$R$ & .67 & & .68 & & .68 & \\
\hline$R^{2}$ & $.45^{* *}$ & & $.46^{* *}$ & & $.47 * *$ & \\
\hline Adjusted $R^{2}$ & .43 & & .44 & & .45 & \\
\hline
\end{tabular}

our findings are consistent with what has been suggested by the social buffering hypothesis (Cohen et al., 1985) because the weak negative association between ikigai and loneliness among those with adequate social support and social

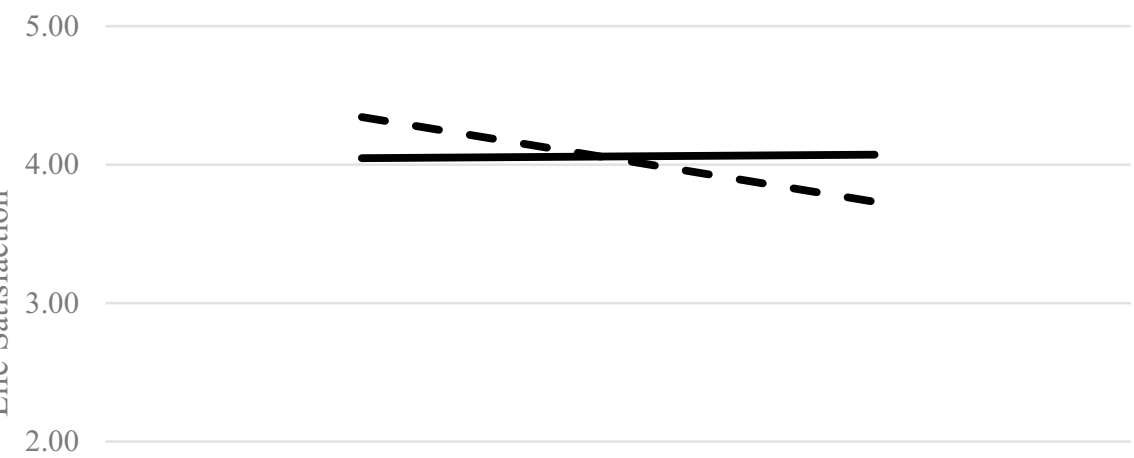

1.00

\section{Lonliness-1SD Loneliness $+1 \mathrm{SD}$}

- SocialSupport-1SD $\longrightarrow$ SocialSupport+1SD

Fig. 1 The interaction between loneliness and social support on life satisfaction 


\subsection{0}

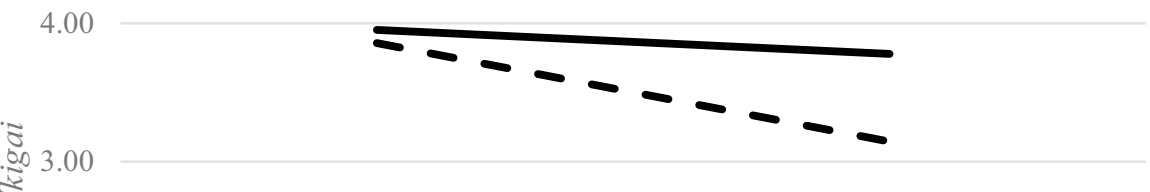

2.00

1.00

Lonliness-1SD

- SocialSupport-1SD

\section{Loneliness $+1 \mathrm{SD}$}

SocialSupport+1SD

Fig. 2 The interaction between loneliness and social support on ikigai

participation suggests that social support and social participation could shield ikigai from stress due to high loneliness. Future longitudinal studies are necessary.

Consistent with our Hypothesis1, the interaction between loneliness and social support on life satisfaction was also significant, and loneliness was negatively associated with life satisfaction only among people with less social support. However, unlike ikigai, life satisfaction did not have a direct negative association with

\subsection{0}

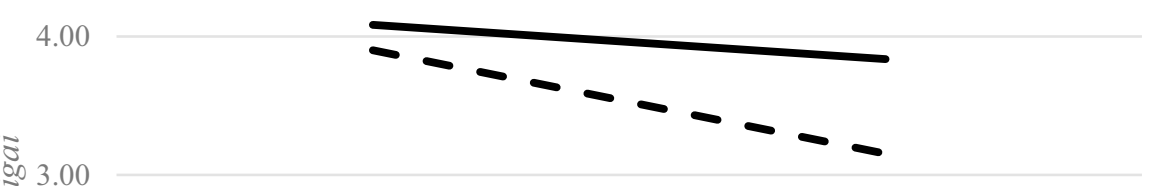

2.00

1.00

\section{Lonliness-1SD}

- SocialParticipation-1SD

\section{Loneliness $+1 \mathrm{SD}$}

SocialParticipation+1SD

Fig. 3 The interaction between loneliness and social participation on ikigai 
loneliness. Thus, although we interpret that loneliness threatens life satisfaction only in people with limited social support, it was unclear whether social support weakens the negative relationship between loneliness and life satisfaction. Moreover, the interaction between loneliness and the frequency of social participation was not significantly related to life satisfaction, which failed to support Hypothesis 2. Therefore, we argue that the social buffering hypothesis is less consistent with the findings on life satisfaction than the findings on ikigai. Thus, we suggest that social support and social participation weaken the relationship between loneliness and well-being, especially ikigai.

One of the strengths of this study is that we investigated two types of wellbeing, life satisfaction, and ikigai, and demonstrated that they have different associations with loneliness and social interaction variables among those in later old age. These differences can be explained by perceived social roles and positive expectations for the future, which are unique to the concept of ikigai (Imai et al., 2009; Shibata, 2002). We interpret that loneliness is negatively related only to ikigai, because loneliness is related to the unique concepts of ikigai, perceived social roles, and positive expectations for the future.

The second strength of this study is that our findings possibly explained why ikigai tends to decline with age among some people in old age, and does not decline among the others. It was demonstrated that ikigai generally tends to decline with age, whereas life satisfaction tends not to decline with age (Kobe Active Aging Research Hub, 2017). It was also demonstrated that approximately $50 \%$ of older adults maintain or increase ikigai with age (Fukuzawa et al., 2019; Kobe Active Aging Research Hub, 2017). The current study's negative association between loneliness and ikigai, and the interaction effects between loneliness and social support and between loneliness and social participation on ikigai can explain these individual differences in ikigai. People who are not lonely might maintain ikigai despite advancing age. Moreover, even lonely people might maintain ikigai if they had adequate social support or frequent social participation through the fulfillment of perceived social roles.

It has been demonstrated that loneliness increases in later old age, and loneliness increases mortality through declined well-being (Pinquart \& Sorensen, 2001; Wang et al., 2020). Because loneliness is a subjective feeling, interventions to reduce loneliness itself might be more difficult than interventions to increase the quantity of social support and opportunities for social participation of those in later old age. The present study suggests that interventions to increase social support and social participation among people in later old age could increase ikigai despite the effects of loneliness among them, and consequently reduce mortality. Longitudinal studies are required to investigate these possibilities.

This study targeted people 75 years and older Japanese, and thus we treated ikigai as a non-Western well-being-related concept. Previous studies suggest other types of non-Western well-being such as interdependent happiness, which is defined as "global, subjective assessment of whether one is interpersonally harmonized with other people, being quiescent, and being ordinary, and connected to the collective way of well-being" (Hitokoto \& Uchida, 2015, p. 214). The concepts of both interdependent happiness and ikigai include satisfaction with 
ones' social interactions. Thus, ikigai and interdependent happiness might have similar relationships with loneliness, social support, and social participation. However, it is also possible that ikigai and interdependent happiness would have different relational patterns with loneliness, social support, and social participation because the concepts of ikigai also include another unique concept, positive expectations for the future (Imai et al., 2009). Future studies including the other types of well-being, such as interdependent well-being, would be necessary.

There are several limitations to this study. Firstly, it was a cross-sectional survey, and longitudinal studies are required in the future to examine how loneliness, social support, and social participation in a given period affect people's well-being over several years. Secondly, we did not assess the details of social participation. Future studies should be designed to identify the types of social participation that are beneficial for maintaining ikigai. Thirdly, the variables of this study, such as social participation, ikigai, and life satisfaction, were measured with single-item scales. Although those single-item scales have been repeatedly used in previous studies, single-item scales might not fully capture individual differences. Future studies with the other several items scales would be necessary to investigate these differences further.

Notwithstanding these limitations, we consider that finding a weak negative association between loneliness and ikigai among people with adequate social support and frequent social participation is important. These findings suggest that interventions for increasing neighborhood social support and opportunities for social participation would maintain ikigai among people 75 years and older. Future studies are expected to identify types of interventions for life satisfaction and ikigai that effectively maintain these aspects of well-being. Moreover, to enrich well-being in older adults, we must clarify if ikigai or life satisfaction is related to individuals' future health status and mortality.

Funding This work was supported by JST RISTEX Grant Number JPMJRX16C1, Japan.

\section{Compliance with Ethical Standards}

Conflict of Interest The authors have no conflicts of interest to declare regarding the findings of this study.

Informed Consent Informed consent was obtained from all individual participants included in the study.

Ethical Treatment of Experimental Subjects (Animals and Humans) No experimental treatment was conducted on either human or animal subjects in this study.

Open Access This article is licensed under a Creative Commons Attribution 4.0 International License, which permits use, sharing, adaptation, distribution and reproduction in any medium or format, as long as you give appropriate credit to the original author(s) and the source, provide a link to the Creative Commons licence, and indicate if changes were made. The images or other third party material in this article are included in the article's Creative Commons licence, unless indicated otherwise in a credit line to the material. If material is not included in the article's Creative Commons licence and your intended use is not permitted by statutory regulation or exceeds the permitted use, you will need to obtain permission directly from the copyright holder. To view a copy of this licence, visit http://creativecommons.org/ licenses/by/4.0/. 


\section{References}

Asher, S. R., \& Weeks, M. S. (2013). Loneliness and Belongingness in the College Years. In R. J. Coplan \& J. C. Bowker (Eds.), The Handbook of Solitude: Psychological Perspectives on Social Isolation Social Withdrawal and Being Alone (pp. 283-301). Hoboken: John Wiley \& Sons Ltd.

Bhagchandani, R. K. (2017). Effect of loneliness on the psychological well-being of college students. International Journal of Social Science and Humanity, 7, 60-64. https://doi.org/10.18178/ijssh.2017.7.1.796

Cacioppo, J. T., Hughes, M. E., Waite, L. J., Hawkley, L. C., \& Thisted, R. A. (2006). Loneliness as a specific risk factor for depressive symptoms: Cross-sectional and longitudinal analyses. Psychology and Aging, 21(1), 140-151. https://doi.org/10.1037/0882-7974.21.1.140

Cheung, F., \& Lucas, R. E. (2014). Assessing the validity of single-item life satisfaction measures: Results from three large samples. Quality of Life Research, 23, 2809-2818. https://doi.org/10.1007/ s11136-014-0726-4

Cohen, J., Cohen, P., West, S. G., \& Aiken, L. S. (2002). Applied Multiple Regression/Correlation Analysis for the Behavioral Sciences (3rd ed.). Lawrence Erlbaum.

Cohen, S., \& Wills, T. A. (1985). Stress, social support, and the buffering hypothesis. Psychological Bulletin, 98(2), 310-357. https://doi.org/10.1037/0033-2909.98.2.310.

Demakakos, P., Nunn, S., \& Nazroo, J. (2006). Loneliness relative deprivation and life satisfaction. In J. Banks, E. Breeze, C. Lessof, \& J. Nazroo (Eds.), Retirement Health and Relationships of The Older Population in England: THE 2004 ENGLISH LONGITUDINAL STUDY OF AGEING (Wave 2) (pp. 297-318). The Institute for Fiscal Studies.

Fujimoto, K., Okada, T., Izumi, T., Mori, K., Yano, E., \& Konishi, M. (2004). Factors defining ikigai of older adults who are living at home. Journal of Health and Welfare Statistics, 51, $24-32$ (In Japanese).

Fukuzawa, A., Katagiri, K., Harada, K., Masumoto, K., Chogahara, M., Kondo, N., \& Okada, S. (2019). A longitudinal study of the moderating effects of social capital on the relationships between changes in human capital and ikigai among Japanese older adults. Asian Journal of Social Psychology, 22, 172-182. https://doi.org/10.1111/ajsp.12353

Gardiner, C., Geldenhuys, G., \& Gott, M. (2018). Interventions to reduce social isolation and loneliness among older people: An integrative review. Health \& Social Care in the Community, 26(2), 147-157. https://doi.org/10.1111/hsc. 12367

Goodwin, R., Cook, O., \& Yung, Y. (2001). Loneliness and life satisfaction among three cultural groups. Personal Relationships, 8(2), 225-230. https://doi.org/10.1111/j.1475-6811.2001.tb00037.x

Harper, R. (2002). The Measurement of Social Capital in The United Kingdom. London, United Kingdom: Office of National Statistics.

Hitokoto, H., \& Uchida, Y. (2015). Interdependent happiness: Theoretical importance and measurement validity. Journal of Happiness Studies, 16, 211-239. https://doi.org/10.1007/s10902-014-9505-8

Imai, T., Osada, H., \& Nishimura, Y. (2009). The structure of IKIGAI concept for retirees over 60 years old: The difference between IKIGAI and subjective well-being. Japanese Journal of Geriatric Psychiatry, 31, 366-377 (In Japanese).

Kapikiran, S. (2013). Loneliness and life satisfaction in Turkish early adolescents: The mediating role of self-esteem and social support. Social Indicators Research, 111(2), 716-632. https://doi.org/10. 1007/s11205-012-0024-X

Kim, J., Lee, S., Chun, S., Han, A., \& Heo, J. (2017). The effects of leisure-time physical activity for optimism life satisfaction psychological well-being and positive affect among older adults with loneliness. Annals of Leisure Research, 20(4), 406-415. https://doi.org/10.1080/11745398.2016.1238308

Kobau, R., Sniezek, J., Zack, M. M., Lucas, R. E., \& Burns, A. (2010). Well-being assessment: An evaluation of well-being scales for public health and population estimates of well-being among US adults. Applied Psychology: Health and Well-Being, 2, 272-297. https://doi.org/10.1111/j.1758-0854.2010. 01035.x

Kobe Active Aging Research Hub. (2017). Active Aging Project Longitudinal Study Report. Kobe University (in Japanese).

Kokubo, T., Iwasaki, F., Tanaka, Y., Tabata, Y., Takayama, T., \& Tamaki, C. (2014). Effects of social interaction on the feeling that life is worth living among elderly island residents. Japanese Society for the Study of Social Welfare, 55(1), 13-22. (in Japanese) 
Kondo, T., \& Kamada, J. (2004). The sex and age differences in the determinants of the feeling that life is worth living among the aged. Japanese Journal of Geriatric Psychiatry, 15, 1281-1290 (in Japanese).

Koivumaa-Honkanen, H., Honkanen, R., Viinamäki, H., Heikkilä, K., Kaprio, J., \& Koskenvuo, M. (2000). Self-reported life satisfaction and 20-year mortality in healthy Finnish adults. American Journal of Epidemiology, 152(10), 983-991. https://doi.org/10.1093/aje/152.10.983

Kono, S., \& Walker, G. (2020). Theorizing the interpersonal aspect of ikigai '(life worth living') among Japanese university students: A mixed-methods approach. International Journal of Wellbeing, 10, 101-123. https://doi.org/10.5502/ijw.v10i2.979

Kumano, M. (2018). On the concept of well-being in Japan: Feeling shiawase as hedonic well-being and feeling ikigai as eudaimonic well-being. Applied Research in Quality of Life, 13, 419-433. https:// doi.org/10.1007/s11482-017-9532-9

Lim, L. L., \& Kua, E. H. (2011). Living alone loneliness and psychological well-being of older persons in Singapore. Current Gerontology and Geriatrics Research, 2011, 673181. https://doi.org/10.1155/ $2011 / 673181$

Liu, L., Gou, Z., \& Zuo, J. (2016). Social support mediates loneliness and depression in elderly people. Journal of Health Psychology, 21(5), 750-758. https://doi.org/10.1177/1359105314536941

Mellor, D., Stokes, M., Firth, L., Hayashi, Y., \& Cummins, R. (2008). Need for belonging relationship satisfaction loneliness and life satisfaction. Personality and Individual Differences, 45(3), 213-218. https://doi.org/10.1016/j.paid.2008.03.020

Nakanishi, N. (1999). 'Ikigai' in older Japanese people. Age and Ageing, 28, 323-324. https://doi.org/10. 1093/ageing/28.3.323

Pinquart, M., \& Sörensen, S. (2000). Influences of socioeconomic status, social network, and competence on subjective well-being in later life: A meta-analysis. Psychology and Aging, 15(2), 187-224. https://doi.org/10.1037/0882-7974.15.2.187

Pinquart, M., \& Sorensen, S. (2001). Influences on loneliness in older adults: A meta-analysis. Basic and Applied Social Psychology, 23, 245-266. https://doi.org/10.1207/S15324834BASP2304_2

Putnam, R. D. (1993). Making Democracy Work: Civic Traditions in Modern Italy. Princeton University Press.

Russell, D., Peplau, L. A., \& Ferguson, M. L. (1978). Developing a measure of loneliness. Journal of Personality Assessment, 42(3), 290-294. https://doi.org/10.1207/s15327752jpa4203_11

Ryan, R., \& Deci, E. L. (2001). On happiness and human potentials: A review of research on hedonic and eudaimonic well-being. Annual Review of Psychology, 52, 141-166. https://doi.org/10.1146/ annurev.psych.52.1.141

Shibata, H. (1998). Required to the elderly. In K. Suzuki (Ed.), Tokyo metropolitan institute of gerontology-successful aging (pp. 47-52). Tokyo: World planning (in Japanese).

Shibata, H. (2002). Constituents of successful aging. Japanese Journal of Geriatrics, 39, 152-154 (In Japanese).

Sone, O., Nakaya, N., Ohmori, K., Shimazu, T., Higashiguchi, M., Kakizaki, M., Kikuchi, N., Kuriyama, S., \& Tsuji, I. (2008). Sense of life worth living (Ikigai) and mortality in Japan: Ohsaki study. Psychosomatic Medicine, 70(6), 709-715. https://doi.org/10.1097/PSY.0b013e31817e7e64

Tanno, K., Sakata, K., Ohsawa, M., Onoda, T., Itai, K., Yaegashi, Y., Tamakoshi, A. \& JACC Study Group. (2009). Associations of ikigai as a positive psychological factor with all-cause mortality and cause-specific mortality among middle-aged and elderly Japanese people: Findings from the Japan Collaborative Cohort Study. Journal of Psychosomatic Research, 67(1), 67-75. https://doi.org/10. 1016/j.jpsychores.2008.10.018.

Uchida, Y., \& Ogihara, Y. (2012). Personal or interpersonal construal of happiness: A cultural psychological perspective. International Journal of Wellbeing, 2, 354-369. https://doi.org/10.5502/ijw.v2.i4.5

Wang, H., Leng, Y., Zhao, E., Fleming, J., \& Brayne, C. (2020). Mortality risk of loneliness in the oldest old over a 10-year follow-up. Aging \& Mental Health, 24, 35-40. https://doi.org/10.1080/13607863. 2018.1510897

Zhang, J., Zhang, J., Zhou, M., \& Yu, N. X. (2018). Neighborhood characteristics and older adults' wellbeing: The roles of sense of community and personal resilience. Social Indicators Research, 137(3), 949-963. https://doi.org/10.1007/s11205-017-1626-0

Publisher's Note Springer Nature remains neutral with regard to jurisdictional claims in published maps and institutional affiliations. 
Ai Fukuzawa received her doctoral degree from the University of Tokyo. She currently works in Institute for Future Initiatives, The University of Tokyo, as a Research Fellow of Japan Society for the Promotion of Science (JSPS). Her recent research interests include factors maintaining or increasing Japanese elderly's well-being (e.g., ikigai, subjective well-being), especially among those with risk factors (e.g., loneliness, low socio-economic status, declined physical health) of declines of their well-being. She focuses on social networks and social participation as the factors maintaining Japanese elderly's well-being from such risks.

Ikuko Sugawara received her doctorial degree from the University of Tokyo. She currently works in the Department of Service Management, Bunri University of Hospitality, as an associate professor. Her recent research interests include association between social networks in community and physical and mental health among community-dwelling late-stage older adults. 